\title{
Using fluorescence lifetime for discriminating detector afterpulsing in fluorescence-correlation spectroscopy
}

\author{
Jörg Enderlein ${ }^{\text {a) }}$ and Ingo Gregor \\ Institute for Biological Information Processing 1 \& 2, Forschungszentrum Jülich, D-52425 Jülich, Germany
}

(Received 3 August 2004; accepted 21 December 2004; published online 15 February 2005)

\begin{abstract}
Fluorescence correlation spectroscopy (FCS) has become an important and widely used technique for many applications in physics, chemistry, and biology. Usually, FCS is measured with sensitive light detectors working in the photon-counting Geiger mode. A common property of such detectors is afterpulsing: the generation of spurious photon detection events after a genuine photon detection. Such afterpulsing causes a significant deviation of the measured autocorrelation function from its true value on a short time scale and can seriously influence derived parameters for fast processes such as triplet-state photophysics. Here, we discuss the impact of afterpulsing on FCS in detail. A new method is developed to eliminate afterpulsing effects by using time-correlated single-photon counting for separating the true fluorescence signal from afterpulsing events. (C) 2005 American Institute of Physics. [DOI: 10.1063/1.1863399]
\end{abstract}

\section{INTRODUCTION}

Fluorescence correlation spectroscopy (FCS) is a relatively old technique, originally introduced by Elson, Magde, and Webb in the early seventies. ${ }^{1-3}$ However, it took nearly two decades for the technique to see a renaissance in single molecule spectroscopy (SMS) after the development of new lasers with high beam quality and temporal stability, lownoise single-photon detectors, and high-quality microscope objectives with nearly perfect imaging quality at high numerical aperture. Achieving values of the detection volume within the range of a few cubic micrometers made the technique applicable for samples at reasonably high concentrations (nM) and enabled reasonably short measurement times (minutes). An excellent and extensive description of FCS can be found in Refs. 4 and 5. For recent reviews see Refs. 6 and 7 and the book in Ref. 8. In FCS, the detected fluorescence intensity is correlated with a time-shifted replica of itself at different values of time shift (lag time). The result is the so-called autocorrelation function (ACF), i.e., the secondorder correlation function of the fluorescence intensity signal. The physical meaning of the ACF is that it is proportional to the probability to detect, on average, a photon at some later time (lag time) if there was a detection event at time zero. This probability is composed of two basically different terms: The two photons detected at time zero and some later lag time can originate from uncorrelated background or from different fluorescing molecules and therefore do not have any physical correlation (provided there is no interaction of the different fluorescing molecules). These events will contribute to a constant offset of the ACF that is completely independent on lag time $\tau$. Alternatively, the two photons originate from one and the same molecule and are therefore physically correlated, leading to a time-dependent

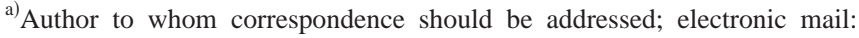
j.enderlein@fz-juelich.de
}

component of the ACF. Thus, the temporal behavior of the $\mathrm{ACF}$ is solely determined by the correlated contributions of individual molecules. In this sense, FCS is a true SMS technique, although the analysis does not explicitly identify single molecule detection events.

On different time scales, the temporal behavior of the autocorrelation function is determined by different properties of the fluorescing molecules: On a nanosecond time scale, photon antibunching can be observed, reflecting the fact that directly after the emission of a photon the molecule needs to get re-excited to be able to emit the next photon, leading to a steep decrease of the ACF towards short times. On a microsecond time scale, the ACF is dominated by triplet state dynamics. If excitation and/or detection are done through polarization filters, the autocorrelation will also show contributions from rotational diffusion dynamics of the molecules. The behavior of the ACF on a microsecond time scale is also strongly influenced by detector afterpulsing. As it will be shown, it is often impossible to clearly distinguish between fast photophysical processes such as triplet state dynamics and detector afterpulsing. On a millisecond to second time scale, the ACF shows a typical decay due to the lateral diffusion of the molecules out of the detection region.

\section{THEORY}

\section{Basic FCS theory}

In an FCS measurement, one records a time trace of fluorescence intensities $I(t)$ detected from a small detection volume and calculates the ACF $g(t)$ at different lag times $t$ as the average

$$
g(t)=\left\langle I\left(t_{0}+t\right) I\left(t_{0}\right)\right\rangle_{t_{0}},
$$

where averaging is done over all possible times $t_{0}$. The shape of this ACF is partially determined by the geometry of the so-called molecule detection function $U(\mathbf{r})(\mathrm{MDF})$ describing the probability to detect a photon when a molecule is 
located at a given position $\mathbf{r}$. Usually, one makes the simplified assumption that this MDF has a three-dimensional Gaussian shape, i.e., that $U(\mathbf{r})$ is proportional to

$$
U(\mathbf{r})=\kappa \exp \left[-\frac{2\left(x^{2}+y^{2}\right)}{a^{2}}-\frac{2 z^{2}}{b^{2}}\right],
$$

where $a$ and $b$ are two characteristic parameters describing the MDF, $\kappa$ is a factor accounting for the overall light detection efficiency of the measurement system as well as the molecules' absorption cross section and fluorescence quantum yield (fluorescence brightness), and $x, y$, and $z$ are Cartesian coordinates, with $z$ along the optical axis. For a solution of diffusing fluorescing molecules with no triplet-state dynamics, the infinite-time limit of the ACF is then given by

$$
\begin{aligned}
\bar{g}_{0}(t)= & \frac{\pi^{3 / 2} \kappa^{2} a^{2} b}{8}\left[\frac{c}{\left(1+4 D t / a^{2}\right) \sqrt{1+4 D t / b^{2}}}\right. \\
& \left.+c^{2} \pi^{3 / 2} a^{2} b\right],
\end{aligned}
$$

where $c$ is the fluorophores' concentration in the sample solution, and $D$ is their diffusion coefficient. If the fluorophores exhibit not only simple singlet ground state/excited state photophysics, but also intersystem crossing from the excited singlet state into the first triplet state with subsequent phosphorescence and return to the singlet ground state, the ACF has additionally an exponentially decaying contribution on the microsecond time scale and can be approximated by ${ }^{9}$

$$
\bar{g}(t)=\left[1+\frac{\phi}{1-\phi} \exp \left(-\frac{t}{\tau}\right)\right] \bar{g}_{0}(t),
$$

where $\phi$ is the average part of molecules residing in the triplet state during their residence time within the detection volume, and $\tau$ is a characteristic decay time depending on intersystem crossing rate, triplet state lifetime, and excitation intensity.

\section{Afterpulsing}

In counting experiments using photomultipliers and avalanche diodes that are operated in the photon-counting (Geiger) mode, several detector nonidealities may play a nonnegligible role (for a detailed discussion, see Ref. 10). The two most common nonidealities are detector dead time and afterpulsing. Detector dead time is caused by a minimum recovery time required for the detector being able to detect the next photon after a previous detection event. Usually, detector dead times are in the range of several tens to hundreds of nanoseconds. They cause the measured ACF to drop towards zero at lag times of the order of the detector dead time. Usually, this time region is of little interest in FCS measurements, and thus, we will not consider dead-time effects further. A more serious effect is detector afterpulsing, meaning that genuine output pulses may be followed by an afterpulse. The origin of afterpulsing and its characteristics depend on the detector type. For photomultipliers the most frequent causes of afterpulsing are ionized atoms of the residual gas that are accelerated towards the photocathode and generate delayed photoelectrons. Other reasons include fluorescence effects of dynodes and luminescence of the residual gas. In semiconductor avalanche diodes a primary photoelectron initiates a chain of ionizations that causes a breakdown pulse at the detector output. Some of the generated charge carriers, however, are temporarily trapped in the junction depletion layer. When these carriers are released by thermal excitation, new free carriers are created that can lead to afterpulses which are correlated with the initial event. The decay times of the traps can be of the order of milliseconds or seconds. The probability of afterpulsing depends on many different parameters like material defects, temperature, and operating conditions of the detector. It can be kept small by reducing the bias voltage below the breakdown voltage after the detection of an output pulse. Hence, the fraction of traps that are released during the time of reduced bias voltage cannot undergo the avalanche multiplication process. In practical applications one has to compromise between high count rates and a tolerable level of afterpulsing. Important for FCS is that afterpulsing is a secondary phenomenon that is correlated to an initial output pulse. Thus, afterpulsing becomes visible as a fast decay of the ACF at lag times comparable with the average time between a genuine detection event and its afterpulse. Let $\varepsilon$ be the overall probability to detect an afterpulse after a genuine detection event. Typical values of $\varepsilon$ range between zero (negligible afterpulsing) and 0.2 . Furthermore, let $p(t)$ denote the temporal probability distribution of detecting an afterpulse at time $t$ after its generating genuine detection event. Typical width of that function is in the range of some microseconds. Taking into account afterpulsing effects, the modified ACF reads

$$
\begin{aligned}
g(t)= & \langle I(t) I(0)\rangle+\varepsilon \int_{0}^{\infty} d t^{\prime} p\left(t^{\prime}\right)\left\langle I\left(t+t^{\prime}\right) I(0)\right\rangle \\
& +\varepsilon \int_{0}^{\infty} d t^{\prime} p\left(t^{\prime}\right)\left\langle I\left(t-t^{\prime}\right) I(0)\right\rangle \\
& +\varepsilon^{2} \int_{0}^{\infty} d t^{\prime} \int_{0}^{t+t^{\prime}} d t^{\prime \prime} p\left(t^{\prime}\right) p\left(t^{\prime \prime}\right)\left\langle I\left(t+t^{\prime}-t^{\prime \prime}\right) I(0)\right\rangle \\
& +\varepsilon p(t)\langle I\rangle,
\end{aligned}
$$

where the first term corresponds to photon detection pairs with no subsequent afterpulses, the second and third terms to photon detection pairs where the first or second detected photon generates an afterpulse, the fourth term to photon detection pairs where both generate afterpulses, and the last term accounts for the correlation between afterpulses and their generating photon detection events. This equation can be rewritten by using the "ideal" ACF $g(t)=\left\langle I\left(t^{\prime}+t\right) I\left(t^{\prime}\right)\right\rangle_{t^{\prime}}$ (with no afterpulsing effects) as

$$
\begin{aligned}
g(t)= & g(t)+\varepsilon \int_{0}^{\infty} d t^{\prime} p\left(t^{\prime}\right) g\left(t+t^{\prime}\right)+\varepsilon \int_{0}^{\infty} d t^{\prime} p\left(t^{\prime}\right) g(\mid t \\
& \left.-t^{\prime} \mid\right)+\varepsilon^{2} \int_{0}^{\infty} d t^{\prime} \int_{0}^{\infty} d t^{\prime \prime} p\left(t^{\prime}\right) p\left(t^{\prime \prime}\right) g\left(\left|t+t^{\prime}-t^{\prime \prime}\right|\right) \\
& +\varepsilon p(t)\langle I\rangle .
\end{aligned}
$$

The first term in this expression is the ideal ACF that one would like to measure, the second through fourth terms are locally averaged versions of this ideal ACF, where $p(t)$ plays 
the role of the local average weight function, and the last term is the temporal correlation between afterpulses and their generating photon detections, thus being directly proportional to $p(t)$. In practice, the last term represents the most significant deviation of the nonideal from the ideal AFC. The second through fourth terms are usually not much different from $g(t)$ as long as the time scale over which $g(t)$ significantly changes is much longer than the width of the function $p(t)$. Unfortunately, typical width of $p(t)$ is in the same temporal range as the characteristic times of triplet-state kinetics. Thus, afterpulsing will mostly interfere with measuring triplet-state photophysics or comparably fast processes via FCS. The usual approach for diminishing the impact of detector afterpulsing on an ACF is to use two separate detectors in two detection channels. Subsequently, the ACF is approximated by cross correlating the signals between the two detectors instead of autocorrelating each detector signal. Because afterpulses from one detector are completely uncorrelated to genuine photon detection events of the other detector, such a cross correlation completely cancels the last term in Eq. (6). However, the second to fourth terms in Eq. (6) remain even after cross correlation and can still lead to a significant deterioration of the ACF at short time scales. Moreover, the necessity to use two detectors always for proper ACF measurements also makes FCS more demanding from a technical point of view.

To get a more quantitative estimation of the impact of afterpulsing on measuring a fast process such as triplet-state dynamics, let us assume that the ideal ACF has the form of Eq. (4) with $g_{0}(t)=$ const. $=\langle I\rangle^{2}$ (no diffusion), and that the afterpulsing probability has an exponential temporal distribution $p=T^{-1} \exp (-t / T)$, a realistic description of the actual afterpulsing behavior of many photon detectors. The modified ACF then takes the explicit form

$$
\begin{aligned}
\widetilde{g}(t)= & (1+\varepsilon)^{2}\langle I\rangle^{2}+\frac{\phi}{1-\phi}\left\{\exp \left(-\frac{t}{\tau}\right)+\frac{\varepsilon(\varepsilon+2)}{1-(T / \tau)^{2}}\right. \\
& \left.\times\left[\exp \left(-\frac{t}{\tau}\right)-\frac{T}{\tau} \exp \left(-\frac{t}{T}\right)\right]\right\}\langle I\rangle^{2} \\
& +\frac{\varepsilon\langle I\rangle}{T} \exp \left(-\frac{t}{T}\right) .
\end{aligned}
$$

Figure 1 shows the behavior of $g(t)$ for different values of $T$. As the figure shows, there can be significant deviations of the measured ACF from its true shape $g(t)$. In particular, both triplet-state dynamics and afterpulsing lead to exponentially decaying terms in the ACF, so that afterpulsing can be easily confused with triplet-state dynamics.

\section{TCSPC-FCS}

Using the information contained in fluorescence lifetime measurement for distinguishing between different fluorescent species in FCS measurements was first proposed in Ref. 11. The same technique also can be used for efficiently eliminating afterpulsing effects in FCS. In a combined fluorescence lifetime and FCS measurement, fluorescence excitation is done with a pulsed laser, and the fluorescence photons are detected on two different times scales (so called time-tagged

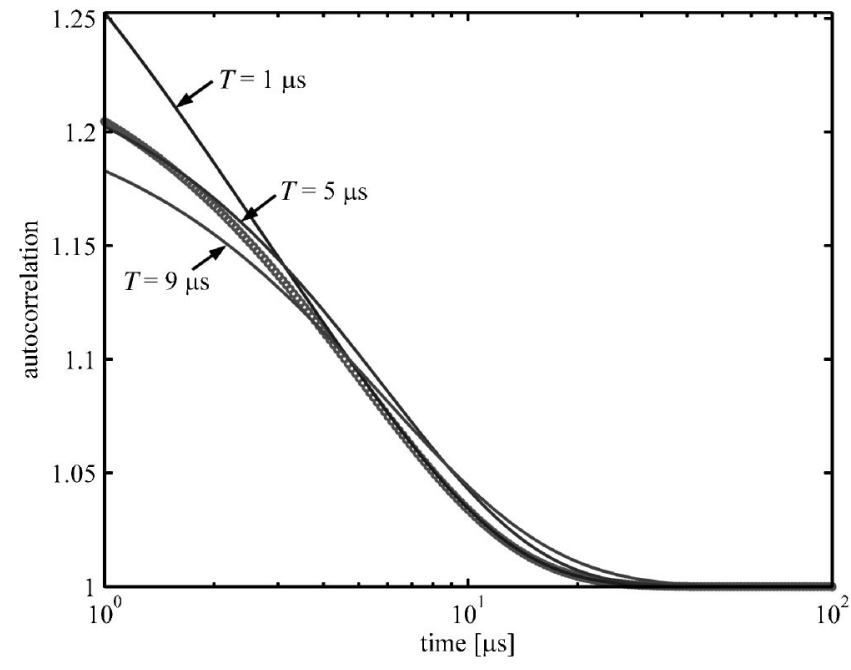

FIG. 1. Impact of afterpulsing on measuring triplet-state dynamics. Open circles show the ideal ACF of an immobile molecule having triplet-state photophysics with triplet-state occupation $\phi=0.2$ and characteristic correlation time $\tau=5 \mu \mathrm{s}$. The solid lines are three ACFs for different afterpulsing time-constants $T$, indicated in $\mu$ s. Total afterpulsing probability of $20 \%$ ( $\varepsilon$ $=0.2$ ) and average count rate of $\langle I\rangle=1 / \mu$ s were assumed.

time-correlated photon counting): on a pico- to nanosecond time scale, where the distance between the exciting laser pulses and the photon detection events is timed (timecorrelated single-photon counting or TCSPC); and on a much larger time-scale between $\sim 100$ nanoseconds up to seconds, where the absolute arrival time of detected photons is recorded, which is subsequently used for calculating the ACF (for details of this kind of time-correlated/time-resolved photon counting, see Refs. 12 and 13). Although afterpulses are correlated with their generating genuine photon detection events, they show negligible picosecond/nanosecond time correlation with respect to the exciting laser pulses: The usual time between subsequent excitation pulses is of the order of a few dozen nanoseconds, whereas the characteristic correlation time between photon detections and subsequent afterpulses is on the order of a few microseconds. Thus, over the time between two laser pulse excitations, the decrease in probability of counting an afterpulse after some photon detection hardly changes, leading to a nearly uniform distribution of afterpulses on the TCSPC time scale. This is the crucial property that is exploited for distinguishing afterpulsing from any fluorescence which quickly decays on the TCSPC time scale.

Thus, let the measured intensity signal $I_{j}$ be

$$
I_{j}(t)=w^{(1)}(t) p_{j}^{(1)}+w^{(2)}(t) p_{j}^{(2)},
$$

where the index $j$ refers to the $j$ th discrete TCSPC time channel used for timing the photon detection events with respect to the exciting laser pulses, $p_{j}^{(1,2)}$ are the normalized fluorescence and afterpulse intensity distributions over these channels, and the $w^{(1,2)}(t)$ are the total intensities of fluorescence and afterpulsing measured on the macroscopic time scale $t$. Looking at Eq. (8), it should be emphasized that two completely different times scales are involved: the macroscopic time scale of $t$, on which the ACF is calculated, and the (discrete) TCSCP time scale labeled by the numbers $j$ of the 
corresponding TCSPC time channel. Thus, one has

$$
g(t)=\left\langle w^{(1)}\left(t_{0}\right) w^{(1)}\left(t_{0}+t\right)\right\rangle_{t_{0}}
$$

and

$$
g(t)=\left\langle\left[w^{(1)}\left(t_{0}\right)+w^{(2)}\left(t_{0}\right)\right]\left[w^{(1)}\left(t_{0}+t\right)+w^{(2)}\left(t_{0}+t\right)\right]\right\rangle_{t_{0}},
$$

where no reference to the TCSPC time scale is present any longer. To recover expression (9) instead of expression (10), one can employ the difference in the temporal distribution of fluorescence and afterpulsing counts over the TCSPC channels. Let us rewrite Eq. (8) in matrix notation as

$$
\mathbf{I}=\mathbf{M} \cdot \mathbf{w},
$$

where $\mathbf{I}$ and $\mathbf{w}$ are column vectors with elements $I_{j}$ and $w^{(k)}$, respectively, and the elements of matrix $\mathbf{M}$ are given by $M_{j k}=p_{j}^{(k)}$. It can be shown ${ }^{14,15}$ that the most likely values of $w^{(k)}(t)$ at every moment $t$ are found by minimizing the quadratic form

$$
(\mathbf{I}-\overline{\mathbf{M}} \mathbf{w})^{T} \cdot \mathbf{V}^{-1} \cdot(\mathbf{I}-\overline{\mathbf{M}} \mathbf{w}),
$$

where $\overline{\mathbf{M}}$ is the average of $\mathbf{M}$ over many excitation cycles, and $\mathbf{V}$ is the covariance matrix given by

$$
\begin{aligned}
\mathbf{V} & =\left\langle(\mathbf{I}-\overline{\mathbf{M}} \mathbf{w}) \cdot(\mathbf{I}-\overline{\mathbf{M}} \mathbf{w})^{T}\right\rangle-\langle(\mathbf{I}-\overline{\mathbf{M}} \mathbf{w})\rangle \cdot\langle(\mathbf{I}-\overline{\mathbf{M}} \mathbf{w})\rangle^{T} \\
& =\operatorname{diag}\langle\mathbf{I}\rangle .
\end{aligned}
$$

Here, triangular brackets denote averaging over an infinite measurement time interval of $t$. In the last equation, it was assumed that the photon detection obeys a Poissonian statistics so that $\left\langle I_{j} I_{k}\right\rangle-\left\langle I_{j}\right\rangle\left\langle I_{k}\right\rangle=\delta_{j k}\left\langle I_{k}\right\rangle$. The solution of the above minimization task is given by using a weighted quasi-inverse matrix operation and has the explicit form

$$
\mathbf{w}=\left[\overline{\mathbf{M}}^{T} \cdot \operatorname{diag}\langle\mathbf{I}\rangle^{-1} \cdot \overline{\mathbf{M}}\right]^{-1} \cdot \overline{\mathbf{M}}^{T} \cdot \operatorname{diag}\langle\mathbf{I}\rangle^{-1} \cdot \mathbf{I}=\mathbf{F} \cdot \mathbf{I} .
$$

Thus, $\mathbf{F}=\left[\overline{\mathbf{M}}^{T} \cdot \operatorname{diag}\langle\mathbf{I}\rangle^{-1} \cdot \overline{\mathbf{M}}\right]^{-1} \cdot \overline{\mathbf{M}}^{T} \cdot \operatorname{diag}\langle\mathbf{I}\rangle^{-1}$ is the soughtafter filter function that recovers $w^{(1)}(t)$ from the measured $I_{j}(t)$

$$
w^{(1)}(t)=\sum_{j=1}^{N} F_{j}^{(1)} I_{j}(t)
$$

(notice that $\mathbf{F}$ is a $2 \times N$ matrix, with elements $F_{j}^{(1,2)}, 1 \leqslant j$ $\leqslant N)$. Finally, the purely fluorescence-generated ACF is calculated as

$$
g(t)=\sum_{j=1}^{N} \sum_{k=1}^{N} F_{j}^{(1)} F_{k}^{(1)}\left\langle I_{j}\left(t_{0}+t\right) I_{k}\left(t_{0}\right)\right\rangle_{t_{0}} .
$$

\section{RESULTS AND DISCUSSION}

FCS-measurements were performed on a dilute aqueous solution of the commercial dye Atto655 (AttoTec), having maximum excitation at $663 \mathrm{~nm}$ and maximum emission at $684 \mathrm{~nm}$ wavelengths. Measurements were performed with a standard confocal epifluorescence setup using a pulsed diode laser with $80 \mathrm{ps}$ pulse with $40 \mathrm{MHz}$ repetition rate and $635 \mathrm{~nm}$ wavelength (PDL 800, PicoQuant) for excitation,

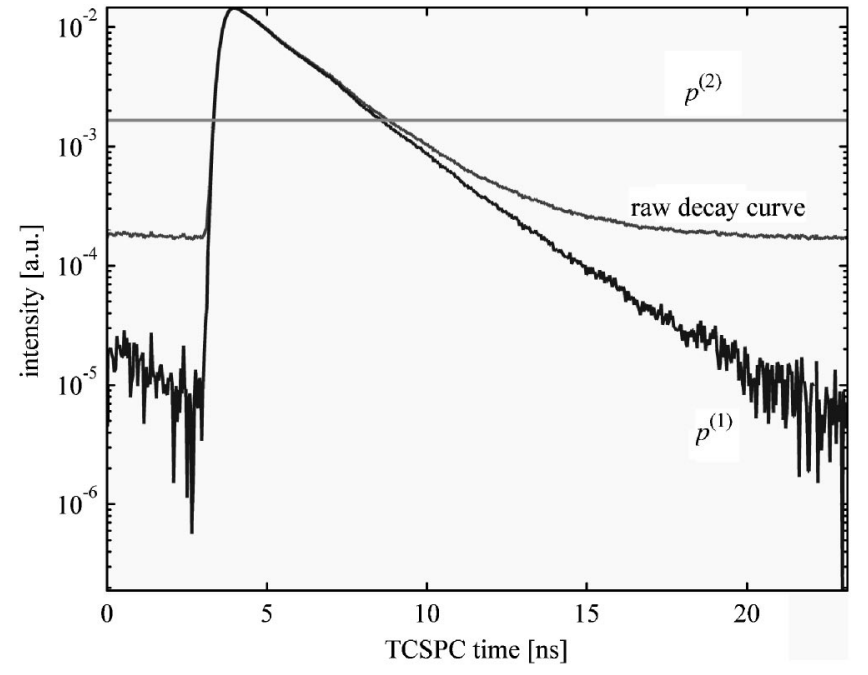

FIG. 2. Measured fluorescence decay curve and deduced fluorescence and afterpulsing TCSPC patterns, $p^{(1)}$ and $p^{(2)}$, respectively.

and a single-photon avalanche diode (SPAD, AQR-14, Perkin-Elmer) for detection. Fast electronics (TimeHarp 200, PicoQuant) was used for recording the detected photons in time-correlated time-tagged recording mode. For a more detailed description of the measurement system and timecorrelated time-tagged photon detection, see Refs. 12 and 13.

Figure 2 shows the fluorescence decay curve, showing the typical fluorescence decay plus a uniform background which is mainly caused by SPAD afterpulsing. For approximating the pure fluorescence decay curve, from the measured curve its minimum value is subtracted, resulting in the nearly monoexponential fluorescence decay curve also shown in Fig. 2. It should be emphasized that the exact character of the fluorescence decay (monoexponential, multiexponential, or other) is completely unimportant; it is only assumed that it decays nearly to zero during one excitation cycle, so that any remaining signal is generated purely by background and afterpulsing. For the subsequent analysis, the measured TCSPC curve minus its minimal value is used, after normalization, as the ideal decay curve $\bar{M}_{j 1}=\bar{p}_{j}^{(1)}$ of the pure fluorescence signal. As the ideal "decay" curve $\bar{M}_{j 2}=\bar{p}_{j}^{(2)}$ of the afterpulsing, a normalized uniform distribution $\bar{p}_{j}^{(2)}=N^{-1}$ is taken, where $N$ is the total number of TCSPC channels. It remains to find an estimate for the average intensity $\left\langle I_{j}\right\rangle$ per TCSPC channel, which is necessary for calculating the covariance matrix $\mathbf{V}$ and thus the filter function $F_{j}^{(1)}$. As a sufficiently good approximation for $\left\langle I_{j}\right\rangle$, one can take the number of measured photons in the $j$ th TCSPC channel over the complete measurement time, so that $\left\langle I_{j}\right\rangle$ is approximated by the raw TCSPC curve as shown in Fig. 2.

For calculating the raw and TCSPC-filtered ACF from the measured time-tagged time-correlated photon detection data, fast algorithms were used as described in Ref. 16. Figure 3 shows the calculated ACFs $g(t)$ and $g(t)$, using either Eq. (1) or the TCSPC-filtered version Eq. (16). The impact of afterpulsing is clearly seen as the fast decay of the unfiltered $\mathrm{ACF}$ at short lag times. In contrast, application of the TCSPC filtering completely eliminates afterpulsing effects, as seen by the total absence of any fast decay of the ACF on a mi- 


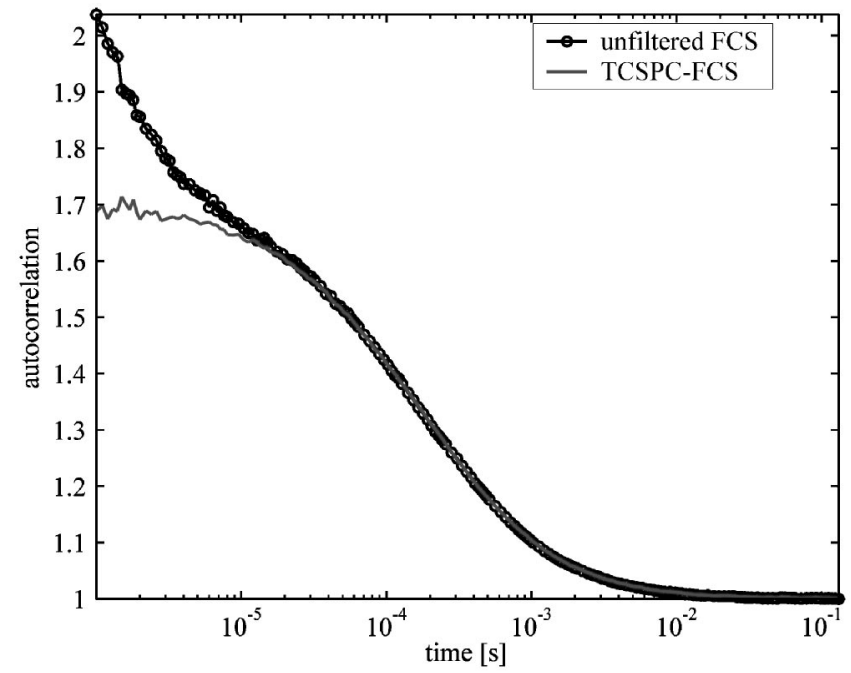

FIG. 3. Unfiltered and TCSPC-filtered ACF for Atto655 in aqueous solution.

crosecond time scale. This was also one reason for using Atto655 as the sample dye: because it was known that this dye has negligible triplet-state photophysics, it is the ideal candidate for demonstrating the efficiency of the TCSPCFCS method.

Although we have used the method of TCSPC-filtered ACF calculation (in short, TCSPC-FCS) here for eliminating afterpulse effects in FCS measurements only, it can be used for separating ACFs for different fluorescent species with sufficiently different fluorescence decay behavior in a single measurement with a single detector (see also Ref. 11). In the case of afterpulse elimination, the method becomes exceptionally simple because no a priori knowledge of any fluorescence decay behavior of the studied sample is necessary: The filter function $F_{j}^{(1)}$ is generated from the same data as those used for the ACF calculation, by simply subtracting from the TCSPC curve its constant (mostly afterpulse generated) background. Thus, the method is completely reference free and straightforward to implement. It eliminates also the impact of any other kind of uniform background, i.e., electronic noise with similarly uncorrelated distribution in TCSPC time space.

\section{ACKNOWLEDGMENTS}

The authors are much obliged to Benjamin Kaupp (IBI-1) and Georg Büldt (IBI-2) for their generous support of our work. Financial support by the Deutsche Forschungsgemeinschaft (Grants EN 297/8-2, FI 841/3-1) is gratefully acknowledged.

${ }^{1}$ D. Magde, E. Elson, and W. W. Webb, Phys. Rev. Lett. 29, 705 (1972).

${ }^{2}$ E. L. Elson and D. Magde, Biopolymers 13, 1 (1974).

${ }^{3}$ D. Magde, E. Elson, and W. W. Webb, Biopolymers 13, 29 (1974).

${ }^{4}$ N. L. Thompson, in Topics in Fluorescence Spectroscopy 1, edited by J. R. Lakowicz (Plenum, New York, 1991), pp. 337-378.

${ }^{5}$ J. Widengren and Ü. Mets, in Single-Molecule Detection in SolutionMethods and Applications, edited by C. Zander, J. Enderlein, and R. A. Keller (Wiley-VCH, Berlin, 2002), pp. 69-95.

${ }^{6}$ P. Schwille, Cell Biochem. Biophys. 34, 383 (2001).

${ }^{7}$ S. T. Hess, S. Huang, A. A. Heikal, and W. W. Webb, Biochemistry 41, 697 (2002).

${ }^{8}$ Fluorescence Correlation Spectroscopy, edited by R. Rigler and E. Elson (Springer, Berlin, 2001).

${ }^{9}$ J. Widengren, Ü. Mets, and R. Rigler, J. Phys. Chem. 99, 13368 (1995).

${ }^{10}$ M. Höbel and J. Ricka, Rev. Sci. Instrum. 65, 2326 (1994).

${ }^{11}$ M. Böhmer, M. Wahl, H. J. Rahn, R. Erdmann, and J. Enderlein, Chem. Phys. Lett. 353, 439 (2002).

${ }^{12}$ M. Böhmer, F. Pampaloni, M. Wahl, H. J. Rahn, R. Erdmann, and J. Enderlein, Rev. Sci. Instrum. 72, 4145 (2001).

${ }^{13}$ M. Böhmer and J. Enderlein, in Ref. 5, pp. 145-183.

${ }^{14}$ G. Leclerc and J. J. Pireaux, J. Electron Spectrosc. Relat. Phenom. 71, 141 (1995).

${ }^{15}$ J. Enderlein and R. Erdmann, Opt. Commun. 134, 371 (1997).

${ }^{16}$ M. Wahl, I. Gregor, M. Patting, and J. Enderlein, Opt. Express 11, 3583 (2003). 